\title{
Omicron extensively but incompletely escapes Pfizer BNT162b2 neutralization
}

https://doi.org/10.1038/s41586-021-04387-1

Received: 14 December 2021

Accepted: 23 December 2021

Published online: 23 December 2021

\section{Open access}

Check for updates

\author{
Sandile Cele ${ }^{1,2}$, Laurelle Jackson', David S. Khoury ${ }^{3}$, Khadija Khan ${ }^{1,2}$, \\ Thandeka Moyo-Gwete ${ }^{4,5}$, Houriiyah Tegally,7, James Emmanuel San ${ }^{6}$, Deborah Cromer ${ }^{3}$, \\ Cathrine Scheepers ${ }^{4,5}$, Daniel G. Amoako ${ }^{2,4}$, Farina Karim 1,2, Mallory Bernstein', Gila Lustig ${ }^{8}$, \\ Derseree Archary ${ }^{8,9}$, Muneerah Smith ${ }^{10}$, Yashica Ganga', Zesuliwe Jule', Kajal Reedoy', \\ Shi-Hsia Hwa ${ }^{1,11}$, Jennifer Giandhari ${ }^{6}$, Jonathan M. Blackburn ${ }^{10,12}$, Bernadett I. Gosnell ${ }^{13}$, \\ Salim S. Abdool Karim ${ }^{8,14}$, Willem Hanekom ${ }^{1,11}$, NGS-SA*, COMMIT-KZN Team*, \\ Anne von Gottberg ${ }^{4,5}$, Jinal N. Bhiman ${ }^{4,5}$, Richard J. Lessells ${ }^{6,8}$, Mahomed-Yunus S. Moosa ${ }^{13}$, \\ Miles P. Davenport ${ }^{3}$, Tulio de Oliveira ${ }^{6,7,8,15}$, Penny L. Moore ${ }^{4,5,8,12}$ \& Alex Sigal ${ }^{1,2,16 \bowtie}$
}

\begin{abstract}
The emergence of the SARS-CoV-2 variant of concern Omicron (Pango lineage B.1.1.529), first identified in Botswana and South Africa, may compromise vaccine effectiveness and lead to re-infections ${ }^{1}$. Here we investigated Omicron escape from neutralization by antibodies from South African individuals vaccinated with Pfizer BNT162b2. We used blood samples taken soon after vaccination from individuals who were vaccinated and previously infected with SARS-CoV-2 or vaccinated with no evidence of previous infection. We isolated and sequence-confirmed live Omicron virus from an infected person and observed that Omicron requires the angiotensin-converting enzyme 2 (ACE2) receptor to infect cells. We compared plasma neutralization of Omicron relative to an ancestral SARS-CoV-2 strain and found that neutralization of ancestral virus was much higher in infected and vaccinated individuals compared with the vaccinated-only participants. However, both groups showed a 22 -fold reduction in vaccine-elicited neutralization by the Omicron variant. Participants who were vaccinated and had previously been infected exhibited residual neutralization of Omicron similar to the level of neutralization of the ancestral virus observed in the vaccination-only group. These data support the notion that reasonable protection against Omicron may be maintained using vaccination approaches.
\end{abstract}

The emergence of the Omicron variant of SARS-CoV-2 in November 2021, first identified in South Africa and Botswana, was first described in South Africa ${ }^{2}$, followed shortly afterwards by confirmed transmission in Hong Kong ${ }^{3}$. Owing to the large number of mutations in the spike protein and elsewhere on the virus (https://covdb.stanford.edu/page/ mutation-viewer/\#omicron), there is concern that this variant will exhibit substantial escape from vaccine-elicited immunity ${ }^{4,5}$. Furthermore, several mutations in the spike receptor-binding domain and S2 fusion domain are predicted to increase transmission ${ }^{5}$.

Here we have used the human lung cell line H1299-ACE2 (Extended Data Fig. 1), which overexpresses the human ACE2 receptor ${ }^{6}$, to both isolate Omicron and test its neutralization by human plasma. We isolated Omicron virus using one passage on H1299-ACE2 cells and a second passage on H1299-ACE2 cells in co-culture with the Vero E6 African green monkey kidney cell line. Sequencing of the isolated virus confirmed it was the Omicron variant bearing the R346K mutation. We observed no mutations introduced in vitro as majority or minority variants (Extended Data Table 1). H1299-ACE2 cells were similar to Vero E6 cells in that they formed infection foci during infection with ancestral D614G and Beta variant viruses; however, the H1299-ACE2 cells formed more foci than unmodified Vero E6 cells (Extended Data Fig. 2a, b). Infection by cell-free Omicron of unmodified Vero E6 cells was inefficient (Extended Data Fig. 2c) and we could not use cell-free Omicron infection in Vero E6 cells to generate a useable virus stock of this isolate (Extended Data Fig. 2d).

We observed that Omicron infected the H1299-ACE2 cells in a concentration-dependent manner but did not infect the parental H1299

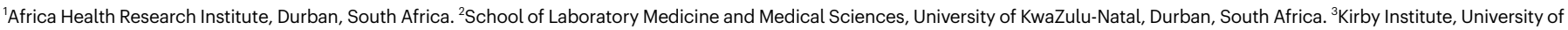
New South Wales, Sydney, New South Wales, Australia. ${ }^{4}$ National Institute for Communicable Diseases of the National Health Laboratory Service, Johannesburg, South Africa. ${ }^{5}$ SA MRC Antibody Immunity Research Unit, School of Pathology, Faculty of Health Sciences, University of the Witwatersrand, Johannesburg, South Africa. ${ }^{6} \mathrm{KwaZulu-Natal} \mathrm{Research} \mathrm{Innovation} \mathrm{and}$

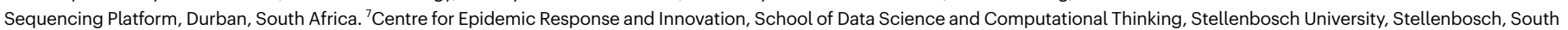
Africa. ${ }^{8}$ Centre for the AIDS Programme of Research in South Africa, Durban, South Africa. ${ }^{9}$ Department of Medical Microbiology, University of KwaZulu-Natal, Durban, South Africa.

${ }^{10}$ Department of Integrative Biomedical Sciences, Faculty of Health Sciences, University of Cape Town, Cape Town, South Africa. "Division of Infection and Immunity, University College London, London, UK. ${ }^{12}$ Institute of Infectious Disease and Molecular Medicine, University of Cape Town, Cape Town, South Africa. ${ }^{13}$ Department of Infectious Diseases, Nelson R. Mandela School of Clinical Medicine, University of KwaZulu-Natal, Durban, South Africa. ${ }^{14}$ Department of Epidemiology, Mailman School of Public Health, Columbia University, New York, NY, USA.

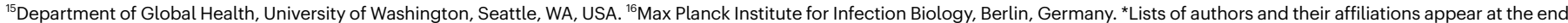
of the paper. ${ }^{凶}$-mail: alex.sigal@ahri.org 


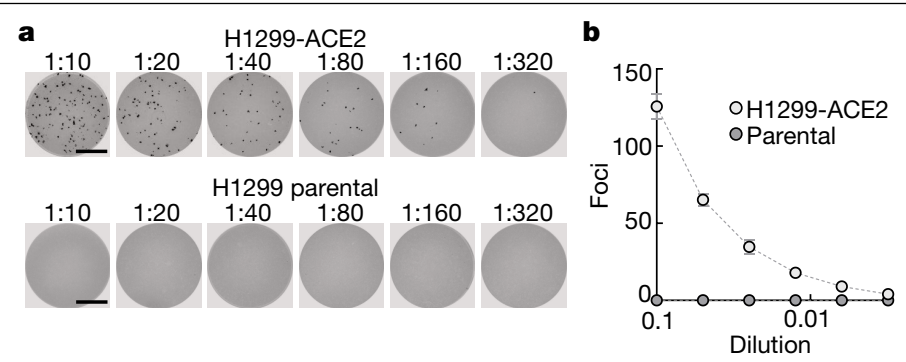

-All o 2xBNT162b2 + previous infection o $2 \times B N T 162 b 2$ o $>50 \%$ neutralization c
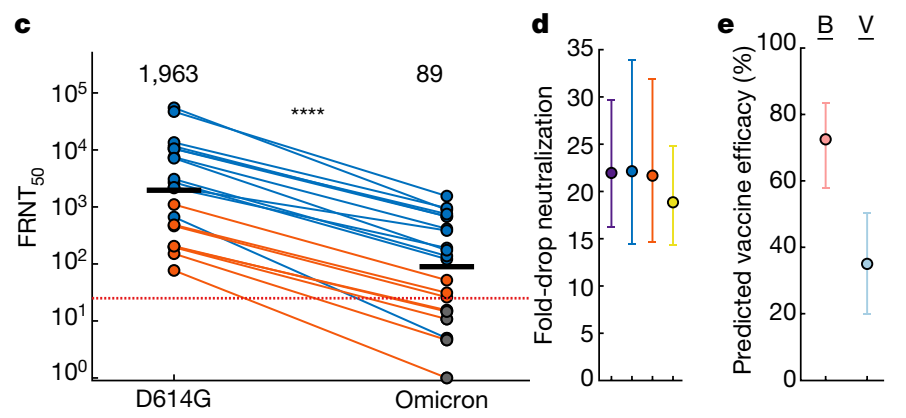

Fig. 1 ACE2 dependence and neutralization of the Omicron variant by Pfizer BNT162b2-elicited immunity.a, Representative images showing infection foci in wells of a multi-well plate with titration of live SARS-CoV-2 Omicron virus on H1299-ACE2 and H1299 parental cells. Numbers above well images denote viral stock dilution. Scale bars, $2 \mathrm{~mm}$. b, Number of foci as a function of Omicron virus stock dilution. Data are mean \pm s.d. of six replicates from two independent experiments. c, Neutralization of Omicron virus compared with D614G ancestral virus by plasma from participants vaccinated with two doses of BNT162b2 and previously SARS-CoV-2 infected (blue) or uninfected (orange). Numbers in black above each virus strain are geometric mean titres (GMT) of the reciprocal plasma dilution $\left(\mathrm{FRNT}_{50}\right.$ ) resulting in $50 \%$ reduction in infection foci. The red horizontal line denotes the most concentrated plasma used. Twenty-one samples were tested from $n=19$ participants in 2 independent experiments $(n=13$ vaccinated and previously infected; $n=6$ vaccinated only). Grey points denote measurements where $50 \%$ neutralization was not achieved with the most concentrated plasma used. $P=4.8 \times 10^{-5}$, Wilcoxon rank-sum test. d, Geometric mean and $95 \%$ confidence interval of the fold change in neutralization between ancestral D614G and Omicron neutralization in plasma. Purple denotes all participants, blue denotes vaccinated individuals with previous SARS-CoV-2 infection, orange denotes vaccinated-only individuals, and yellow denotes all participants excluding those in whom $50 \%$ neutralization was not achieved.e, Mean predicted vaccine efficacy and $95 \%$ confidence intervals against symptomatic infection with Omicron using data from previous randomized controlled trials and the 22-fold difference between D614G and Omicron observed in this study ${ }^{17,18}$. Predictions are for vaccinated and boosted (B, red) or vaccinated-only ( $\mathrm{V}$, blue) individuals.

cells, indicating that human ACE2 is required for Omicronentry(Fig.1a,b). We then tested the ability of plasma from individuals vaccinated with BNT162b2 to neutralize Omicron versus ancestral D614G virus in a live virus neutralization assay. We tested plasma samples taken from 19 individuals after they had received 2 doses of BNT162b2 (Extended Data Tables 2,3), 6 of whom had no previous record of SARS-CoV-2 infection or detectable SARS-CoV-2 nucleocapsid antibodies indicative of previous infection (Methods). We also tested samples from a later time point for two of the vaccinated-only participants (Extended Data Table 3). The previously infected and vaccinated participants were infected with either ancestral SARS-CoV-2 strains or the Delta variant (Extended Data Table 3). To quantify neutralization in the live virus neutralization assay, we calculated the focus reduction neutralization test value $\left(\mathrm{FRNT}_{50}\right.$, the inverse of the plasma dilution required for a $50 \%$ reduction in infection focus number).
Consistent with previous studies ${ }^{7-9}$, we observed that individuals who were vaccinated and had previously been infected exhibited higher neutralization capacity for ancestral virus relative to those who were vaccinated only (Fig. 1c). For all participants, the ability to neutralize Omicron was lower than for ancestral virus (Fig. 1c). The geometric mean titre (GMT) FRNT $_{50}$ for all participants was 1,963 for D614G and 89 for Omicron, a 22-fold difference (95\% confidence interval 16-30) (Fig. 1d); the fold drop was the same for individuals who were vaccinated and had previously been infected (95\% confidence interval 16-34) and in the vaccinated-only group (95\% confidence interval 15-32) (Fig.1d). Six of the samples showed fitted values for $50 \%$ Omicron neutralization that corresponded to a plasma concentration higher than the most concentrated plasma tested (a 1:25 dilution). This included the two samples collected at a later time point after vaccination, one of which showed a complete knockout of neutralization activity with Omicron (Fig. 1c, Extended Data Table 3). Excluding these 6 values from the analysis reduced the difference in $\mathrm{GMT} \mathrm{FRNT}_{50}$ between D614G and Omicron to 19-fold (95\% confidence interval 14-25), well within the $95 \%$ confidence intervals of the fold differences for the raw values (Fig.1d). Of note, Omicron virus neutralization by samples from individuals who were previously infected and vaccinated was similar to D614G neutralization by samples from participants vaccinated with two doses of BNT162b2 but not previously infected (Fig. 1c). GMT FRNT ${ }_{50}$ for Omicron in the previously infected and vaccinated group was 305 (95\% confidence interval 134-695), whereas GMT FRNT for $_{50}$ foncestral virus in the vaccinated-only group was 263 (95\% confidence interval 147-472).

We compared these results with neutralization of the Beta variant $t^{6,10-16}$ using Beta and D614G virus infection of H1299-ACE2 (Extended Data Fig. 3a) and Vero E6 (Extended Data Fig. 3b) cells. The fold difference relative to the ancestral D614G virus was 4.3 for H1299-ACE2 cells and 5.0 for Vero E6 cells. Thus, results from these two cell lines indicated that Omicron exhibited approximately fourfold greater escape relative to Beta in our assays.

This study was not designed to reliably evaluate vaccine efficacy or protection from severe disease. However, a prediction of vaccine efficacy after a 22-fold drop in neutralization can be made in individuals vaccinated with BNT162b2 and individuals who were both vaccinated and boosted on the basis of data from randomized control trials using a model relating neutralization level to vaccine efficacy ${ }^{17,18}$. Using this model and the differences in neutralization between Omicron and other SARS-CoV-2 strains (Methods), we predict a vaccine efficacy for preventing symptomatic infection by Omicron of $73 \%$ (95\% confidence interval 58-83\%) for vaccinated and boosted individuals, and 35\% (95\% confidence interval 20-50\%) for vaccinated-only individuals; this suggests that Omicron compromises the ability of the vaccine to protect against infection in individuals in the vaccinated-only group but not in vaccinated and boosted individuals (Fig. 1e). We note that these predictions are similar to reports of actual vaccine efficacy in the $\mathrm{UK}^{19}$.

Shortly after we released these findings, several other groups have reported similar results ${ }^{3,20-23}$ including Pfizer-BioNTech (https://www. businesswire.com/news/home/20211208005542/en/). These results mirror ours, with large reductions in neutralization of Omicron compared with ancestral virus by vaccine-elicited immunity, neutralizing monoclonal antibodies and plasma from convalescent individuals previously infected with other variants. Notably, the Pfizer-BioNTech study reports that boosting seems to increase neutralization breadth, which reduces the escape by Omicron relative to ancestral virus; these results have been validated independently ${ }^{21}$. Unlike what was reported for boosting, we did not observe a lower fold drop in previously vaccinated and infected relative to the vaccinated-only participants in this study.

Limitations of this study include the presence of an R346K substitution in our virus stock. This putative escape mutation ${ }^{24}$, which may confer moderate antibody resistance (https://jbloomlab.github.io/ SARS2_RBD_Ab_escape_maps/escape-calc/), is not found in the majority 
of Omicron genomes. In addition, the timing of sample collection soon after vaccination (Supplementary Tables 2,3) does not account for the waning of neutralization capacity ${ }^{25,26}$.

So far, a milder course of Omicron infection has been observed in South Africa relative to previous infection waves in terms of reported numbers of patients in intensive care units and needing ventilation ${ }^{27}$. Although there may be other unidentified contributing factors that lower pathogenicity ${ }^{28}$, pre-existing immunity would be expected to be higher in the Omicron wave because of vaccination as well as immunity elicited by previous infection during one of three preceding infection waves in South Africa ${ }^{28}$. Therefore, the incomplete Omicron escape from previous immunity described here may be an important factor accounting for the milder course of infection. Despite the extensive neutralization escape of Omicron, residual neutralization levels may still be sufficient to protect from severe disease $\mathrm{e}^{17,18}$. Other facets of the adaptive immune response elicited by vaccination and previous infection may increase protection. Furthermore, we observed that vaccination combined with previous infection elicits similar neutralization capacity against Omicron as vaccination without previous infection elicits against ancestral virus. This indicates that protection from symptomatic Omicron infection may occur when vaccination is combined with previous infection or boosting. This may explain why Pfizer BNT162b2 vaccination has been shown to substantially decrease the risk of hospital admission caused by Omicron infection in South Africa ${ }^{29}$ and supports the use of further vaccination and boosting to combat Omicron.

\section{Online content}

Any methods, additional references, Nature Research reporting summaries, source data, extended data, supplementary information, acknowledgements, peer review information; details of author contributions and competing interests; and statements of data and code availability are available at https://doi.org/10.1038/s41586-021-04387-1.

1. Pulliam, J.R. C. et al. Increased risk of SARS-CoV-2 reinfection associated with emergence of the Omicron variant in South Africa. Preprint at https://doi.org/ 10.1101/2021.11.11.21266068 (2021).

2. Viana, R. et al. Rapid epidemic expansion of the SARS-CoV-2 Omicron variant in southern Africa. Nature, https://doi.org/10.1038/s41586-022-04411-y (2022).

3. Lu, L. et al. Neutralization of SARS-CoV-2 Omicron variant by sera from BNT162b2 or Coronavac vaccine recipients. Clin. Infect. Dis. https://doi.org/10.1093/cid/ciab1041 (2021).

4. Harvey, W. T. et al. SARS-CoV-2 variants, spike mutations and immune escape. Nat. Rev. Microbiol. 19, 409-424 (2021).

5. Tao, K. et al. The biological and clinical significance of emerging SARS-CoV-2 variants. Nat. Rev. Genet. 22, 757-773 (2021).

6. Cele, S. et al. Escape of SARS-CoV-2 501Y.V2 from neutralization by convalescent plasma. Nature 593, 142-146 (2021).

7. Keeton, R. et al. Prior infection with SARS-CoV-2 boosts and broadens Ad26.COV2.S immunogenicity in a variant-dependent manner. Cell Host Microbe 29, 1611-1619.e1615 (2021).

8. Stamatatos, L. et al. mRNA vaccination boosts cross-variant neutralizing antibodies elicited by SARS-CoV-2 infection. Science 372, 1413-1418 (2021).

9. Ebinger, J. E. et al. Antibody responses to the BNT162b2 mRNA vaccine in individuals previously infected with SARS-CoV-2. Nat. Med. 27, 981-984 (2021).

10. Tegally, H. et al. Detection of a SARS-CoV-2 variant of concern in South Africa. Nature 592, 438-443 (2021)

11. Garcia-Beltran, W. F. et al. Multiple SARS-CoV-2 variants escape neutralization by vaccine-induced humoral immunity. Cell 184, 2372-2383.e2379 (2021).

12. Wibmer, C. K. et al. SARS-CoV-2 501Y.V2 escapes neutralization by South African COVID-19 donor plasma. Nat. Med. 27, 622-625 (2021).

13. Zhou, D. et al. Evidence of escape of SARS-CoV-2 variant B.1.351 from natural and vaccine-induced sera. Cell 184, 2348-2361.e2346 (2021).

14. Planas, D. et al. Sensitivity of infectious SARS-CoV-2 B.1.1.7 and B.1.351 variants to neutralizing antibodies. Nat. Med. 27, 917-924 (2021).

15. Wang, P. et al. Antibody resistance of SARS-CoV-2 variants B.1.351 and B.1.1.7. Nature 593, 130-135 (2021)

16. Wang, Z. et al. mRNA vaccine-elicited antibodies to SARS-CoV-2 and circulating variants. Nature 592, 616-622 (2021)

17. Cromer, D. et al. Neutralising antibody titres as predictors of protection against SARS-CoV-2 variants and the impact of boosting: a meta-analysis. Lancet Microbe $\mathbf{3}$, e52-e61 (2021).
18. Khoury, D. S. et al. Neutralizing antibody levels are highly predictive of immune protection from symptomatic SARS-CoV-2 infection. Nat. Med. 3, 1205-1211 (2021).

19. Andrews, N. et al. Effectiveness of COVID-19 vaccines against the Omicron (B.1.1.529) variant of concern. Preprint at https://doi.org/10.1101/2021.12.14.21267615 (2021).

20. Wilhelm, A. et al. Reduced neutralization of SARS-CoV-2 Omicron variant by vaccine sera and monoclonal antibodies. Preprint at https://doi.org/10.1101/2021.12.07.21267432 (2021).

21. Garcia-Beltran, W. F. et al. mRNA-based COVID-19 vaccine boosters induce neutralizing immunity against SARS-CoV-2 Omicron variant. Preprint at https://doi.org/10.1101/ 2021.12.14.21267615 (2021).

22. Rössler, A., Riepler, L., Bante, D., Laer, D. V. \& Kimpel, J. SARS-CoV-2 B.1.1.529 variant (Omicron) evades neutralization by sera from vaccinated and convalescent individuals. Preprint at https://doi.org/10.1101/2021.12.08.21267491 (2021).

23. Cao, Y. et al. Omicron escapes the majority of existing SARS-CoV-2 neutralizing antibodies. Nature, https://doi.org/10.1038/s41586-021-04385-3 (2021).

24. Weisblum, Y. et al. Escape from neutralizing antibodies by SARS-CoV-2 spike protein variants. eLife 9, e61312 (2020).

25. Goldberg, Y. et al. Waning immunity after the BNT162b2 vaccine in Israel. N. Engl. J. Med. 385, e85 (2021).

26. Chemaitelly, H. et al. Waning of BNT162b2 vaccine protection against SARS-CoV-2 infection in Qatar. N. Engl. J. Med. 385, e83 (2021).

27. Abdullah, F. et al. Decreased severity of disease during the first global Omicron variant COVID-19 outbreak in a large hospital in Tshwane, South Africa. Int. J. Infect. Dis. 116, 38-42 (2022).

28. Cele, S. et al. SARS-CoV-2 evolved during advanced HIV disease immunosuppression has Beta-like escape of vaccine and Delta infection elicited immunity. Preprint at https://doi.org/10.1101/2021.09.14.21263564 (2021).

29. Collie S., Champion J., Moultrie H., Bekker L. G. \& Gray G. Effectiveness of BNT162b2 vaccine against Omicron variant in South Africa. N. Engl. J. Med. 386, 494-496 (2022).

Publisher's note Springer Nature remains neutral with regard to jurisdictional claims in published maps and institutional affiliations.

Open Access This article is licensed under a Creative Commons Attribution 4.0 International License, which permits use, sharing, adaptation, distribution and reproduction in any medium or format, as long as you give appropriate credit to the original author(s) and the source, provide a link to the Creative Commons license, and indicate if changes were made. The images or other third party material in this article are included in the article's Creative Commons license, unless indicated otherwise in a credit line to the material. If material is not included in the article's Creative Commons license and your intended use is not permitted by statutory regulation or exceeds the permitted use, you will need to obtain permission directly from the copyright holder. To view a copy of this license visit http://creativecommons.org/licenses/by/4.0/.

(c) The Author(s) 2021

NGS-SA

Mary-Ann Davies ${ }^{17}$, Marvin Hsiao ${ }^{18}$, Darren Martin ${ }^{12,19}$, Koleka Mlisana $^{20,21}$, Constantinos Kurt Wibmer ${ }^{4}$, Carolyn Williamson ${ }^{4,12,22}$ \& Denis York ${ }^{23}$

${ }^{17}$ Center for Infectious Disease Epidemiology and Research, School of Public Health and Family Medicine, University of Cape Town, Cape Town, South Africa. ${ }^{18}$ University of Cape Town/Groote Schuur Complex of the National Health Laboratory Service (NHLS), University of Cape Town, Cape Town, South Africa. ${ }^{19}$ Division of Computational Biology, Department of Integrative Biomedical Sciences, University of Cape Town, Cape Town, South Africa.

${ }^{20}$ Medical Microbiology Department, University of KwaZulu-Natal, Durban, South Africa. ${ }^{21}$ National Health Laboratory Services (NHLS), Durban, South Africa. ${ }^{22}$ Wellcome Centre for Infectious Diseases Research in Africa, University of Cape Town, Cape Town, South Africa. ${ }^{23}$ Molecular Diagnostics Services, Durban, South Africa.

\section{COMMIT-KZN Team}

Rohen Harrichandparsad ${ }^{24}$, Kobus Herbst ${ }^{1,25}$, Prakash Jeena ${ }^{26}$, Thandeka Khoza ${ }^{1}$ Henrik Kløverpris $^{1,27}$, Alasdair Leslie ${ }^{111}$, Rajhmun Madansein ${ }^{28}{ }^{2}$, Nombulelo Magula ${ }^{29}$, Nithendra Manickchund ${ }^{13}$, Mohlopheni Marakalala ${ }^{111}$, Matilda Mazibuko', Mosa Moshabela ${ }^{30}$, Ntombifuthi Mthabela', Kogie Naidoo ${ }^{8}$, Zaza Ndhlovu ${ }^{131}$, Thumbi Ndung' ' ${ }^{1,16,31,32}$, Nokuthula Ngcobo', Kennedy Nyamande ${ }^{33}$, Vinod Patel ${ }^{34}$, Theresa Smit', Adrie Steyn ${ }^{1,35}$ \& Emily Wong ${ }^{1,35}$

${ }^{24}$ Department of Neurosurgery, University of KwaZulu-Natal, Durban, South Africa. ${ }^{25}$ South African Population Research Infrastructure Network, Durban, South Africa. ${ }^{26}$ Department of Paediatrics and Child Health, University of KwaZulu-Natal, Durban, South Africa. ${ }^{27}$ Department of Immunology and Microbiology, University of Copenhagen, Copenhagen, Denmark. ${ }^{28}$ Department of Cardiothoracic Surgery, University of KwaZulu-Natal, Durban, South Africa. ${ }^{29}$ Department of Medicine, King Edward VIII Hospital and University of KwaZulu Natal, Durban, South Africa. ${ }^{30}$ College of Health Sciences, University of KwaZulu-Natal, Durban, South Africa. ${ }^{31}$ Ragon Institute of MGH, MIT and Harvard, Boston, MA, USA. ${ }^{32} \mathrm{HIV}$ Pathogenesis Programme, The Doris Duke Medical Research Institute, University of KwaZulu-Natal, Durban, South Africa. ${ }^{33}$ Department of Pulmonology and Critical Care, University of KwaZulu-Natal, Durban, South Africa. ${ }^{34}$ Department of Neurology, University of KwaZulu-Natal, Durban, South Africa.

${ }^{35}$ Division of Infectious Diseases, University of Alabama at Birmingham, Birmingham, AL, USA. 


\section{Methods}

\section{Whole-genome sequencing, genome assembly and phylogenetic analysis}

cDNA synthesis was performed on the extracted RNA using random primers followed by gene-specific multiplex PCR using the ARTIC V.3 protocol (https://www.protocols.io/view/covid-19-artic-v3-ill umina-library-construction-an-bibtkann). In brief, extracted RNA was converted to cDNA using the Superscript IV First Strand synthesis system (Life Technologies) and random hexamer primers. SARS-CoV-2 whole-genome amplification was performed by multiplex PCR using primers designed using Primal Scheme (http://primal.zibraproject. org/) to generate 400-bp amplicons with an overlap of $70 \mathrm{bp}$ that covers the 30-kb SARS-CoV-2 genome. PCR products were cleaned up using AmpureXP purification beads (Beckman Coulter) and quantified using the Qubit dsDNA High Sensitivity assay on the Qubit 4.0 instrument (Life Technologies). We then used the Illumina Nextera Flex DNA Library Prep kit according to the manufacturer's protocol to prepare indexed paired-end libraries of genomic DNA. Sequencing libraries were normalized to $4 \mathrm{nM}$, pooled and denatured with $0.2 \mathrm{~N}$ sodium acetate. Then, a 12-pM sample library was spiked with $1 \%$ PhiX (a PhiX Control v.3 adaptor-ligated library was used as a control). We sequenced libraries on a 500-cycle v.2 MiSeq Reagent Kit on the Illumina MiSeq instrument (Illumina). We assembled paired-end fastq reads using Genome Detective 1.126 (https://www.genomedetective.com) and the Coronavirus Typing Tool. We polished the initial assembly obtained from Genome Detective by aligning mapped reads to the reference sequences and filtering out low-quality mutations using the bcftools 1.7-2 mpileup method. Mutations were confirmed visually with BAM files using Geneious software (Biomatters). P2 stock was sequenced and confirmed Omicron with the following substitutions: E:T9I, M:D3G, M:Q19E, M:A63T, N:P13L, N:R203K, N:G204R, ORF1a:K856R, ORF1a:L2084I, ORF1a:A2710T, ORF1a:T3255I, ORF1a:P3395H, ORF1a:I3758V, ORF1b:P314L, ORF1b:I1566V, ORF9b:P10S, S:A67V, S:T95I, S:Y145D, S:L212I, S:G339D, S:R346K, S:S371L, S:S373P, S:S375F, S:K417N, S:N440K, S:G446S, S:S477N, S:T478K, S:E484A, S:Q493R, S:G496S, S:Q498R, S:N501Y, S:Y505H, S:T547K, S:D614G, S:H655Y, S:N679K, S:P681H, S:N764K, S:D796Y, S:N856K, S:Q954H, S:N969K and S:L981F. Deletions: N:E31, N:R32, N:S33, ORF1a:S2083, ORF1a:L3674, ORF1a:S3675, ORF1a:G3676, ORF9b:E27, ORF9b:N28, ORF9b:A29, S:H69, S:V70, S:G142, S:V143, S:Y144 and S:N211. The sequence was deposited at GISAID under accession EPI_ISL_7358094.

\section{SARS-CoV-2 nucleocapsid enzyme-linked immunosorbent assay (ELISA)}

Nucleocapsid protein $\left(2 \mu \mathrm{g} \mathrm{ml}^{-1}\right)$ (Biotech Africa; catalogue (cat.) no. BA25-P) was used to coat 96-well, high-binding plates and incubated overnight at $4{ }^{\circ} \mathrm{C}$. The plates were incubated in a blocking buffer consisting of $5 \%$ skimmed milk powder, $0.05 \%$ Tween $20,1 \times$ PBS. Plasma samples were diluted to a 1:100 dilution in a blocking buffer and added to the plates. Horseradish peroxidase (HRP)-conjugated IgG secondary antibody was diluted to 1:3,000 in blocking buffer and added to the plates followed by tetramethylbenzidine (TMB) peroxidase substrate (Thermo Fisher Scientific). Upon stopping the reaction with $1 \mathrm{M} \mathrm{H}_{2} \mathrm{SO}_{4}$, absorbance was measured at a 450-nm wavelength.

\section{Cells}

Vero E6 cells (ATCC CRL-1586, obtained from Cellonex) were propagated in complete growth medium consisting of Dulbecco's modified Eagle medium (DMEM) with $10 \%$ fetal bovine serum (Hyclone) containing $10 \mathrm{mM}$ of HEPES, $1 \mathrm{mM}$ sodium pyruvate, $2 \mathrm{mM}$-glutamine and $0.1 \mathrm{mM}$ nonessential amino acids (Sigma-Aldrich). Vero E6 cells were passaged every 3-4 days. $\mathrm{H} 1299$ cell lines were propagated in growth medium consisting of complete Roswell Park Memorial Institute (RPMI) 1640 medium with $10 \%$ fetal bovine serum containing $10 \mathrm{mM}$ of HEPES, $1 \mathrm{mM}$ sodium pyruvate, $2 \mathrm{mM}$ L-glutamine and $0.1 \mathrm{mM}$ nonessential amino acids. $\mathrm{H} 1299$ cells were passaged every second day. The H1299-E3 (H1299-ACE2, clone E3) cell line was derived from H1299 (CRL-5803) as described in previous work ${ }^{6}$ and Supplementary Fig. 1. In brief, vesicular stomatitis virus G glycoprotein (VSVG) pseudotyped lentivirus containing ACE2 was used to spinfect H1299 cells. ACE-2 transduced $\mathrm{H} 1299$ cells (containing an endogenously yellow fluorescent protein labelled histone $\mathrm{H} 2 \mathrm{AZ}$ gene $^{30}$ ) were then subcloned at single-cell density in 96-well plates (Eppendorf) in conditioned medium derived from confluent cells. After 3 weeks, wells were detached using a $0.25 \%$ trypsin-EDTA solution (Gibco) and plated in 2 replicate plates, where the first plate was used to determine infectivity and the second was stock. The first plate was screened for the fraction of mCherry-positive cells per cell clone upon infection with a SARS-CoV-2 mCherry expressing spike pseudotyped lentiviral vector. Screening was performed using a Metamorph-controlled (Molecular Devices) Nikon TiE motorized microscope (Nikon) with a $20 \times, 0.75 \mathrm{NA}$ phase objective, 561-nm laser line, and 607-nm emission filter (Semrock). Images were captured using an 888 EMCCD camera (Andor). The clone with the highest fraction of mCherry expression was expanded from the stock plate and denoted H1299-E3. Infectivity was confirmed with mCherry expressing lentivirus by flow cytometry using a BD Fortessa instrument and analysed using BD FACSDiva Software (BD Biosciences). This clone was used in the outgrowth and focus forming assay. Cell lines have not been authenticated. The cell lines have been tested for mycoplasma contamination and are mycoplasma negative.

\section{Virus expansion}

All work with live virus was performed in biosafety level 3 containment using protocols for SARS-CoV-2 approved by the Africa Health Research Institute Biosafety Committee. ACE2-expressing H1299-E3 cells were seeded at $4.5 \times 10^{5}$ cells in a well on a 6 -well plate and incubated for 18-20 h. After one DPBS wash, the sub-confluent cell monolayer was inoculated with $500 \mu$ l universal transport medium diluted 1:1 with growth medium filtered through a $0.45-\mu \mathrm{m}$ filter. Cells were incubated for $1 \mathrm{~h}$. Wells were then filled with $3 \mathrm{ml}$ complete growth medium. After 4 days of infection (completion of passage 1(P1)), cells were trypsinized, centrifuged at $300 \mathrm{~g}$ for $3 \mathrm{~min}$ and resuspended in $4 \mathrm{ml}$ growth medium. Then, $2 \mathrm{ml}$ was added to Vero $\mathrm{E} 6$ cells that had been seeded at $2 \times 10^{5}$ cells per $\mathrm{ml}, 5 \mathrm{ml}$ total, $18-20 \mathrm{~h}$ earlier in a T25 flask (approximately 1:8 donor-to-target cell dilution ratio) for cell-to-cell infection. The co-culture of ACE2-expressing H1299-E3 and Vero E6 cells was incubated for $1 \mathrm{~h}$ and $7 \mathrm{ml}$ of complete growth medium was added to the flask and incubated for 4 days. The viral supernatant (passage 2(P2) stock) was used for experiments. Further optimization of the viral outgrowth protocol used for subsequent Omicron isolates showed that addition of $4 \mathrm{ml}$ instead of $2 \mathrm{ml}$ of infected H1299-E3 cells to Vero E6 cells that had been seeded at $2 \times 10^{5}$ cells per $\mathrm{ml}, 20 \mathrm{ml}$ total, 18-20 h earlier in a T75 flask gave P2 stocks with substantially higher titres that could detectably infect Vero E6 cells. The Omicron virus isolate is available from the authors contingent on verification that it will be received and used in a biosafety level 3 facility.

\section{Live virus neutralization assay}

H1299-E3 cells were plated in a 96-well plate (Corning) at 30,000 cells per well 1 day before infection. Plasma was separated from EDTA-anticoagulated blood by centrifugation at $500 \mathrm{~g}$ for $10 \mathrm{~min}$ and stored at $-80^{\circ} \mathrm{C}$. Aliquots of plasma samples were heat-inactivated at $56^{\circ} \mathrm{C}$ for $30 \mathrm{~min}$ and clarified by centrifugation at $10,000 \mathrm{~g}$ for $5 \mathrm{~min}$. Virus stocks were used at approximately $50-100$ focus-forming units per microwell and added to diluted plasma. Antibody-virus mixtures were incubated for $1 \mathrm{~h}$ at $37^{\circ} \mathrm{C}, 5 \% \mathrm{CO}_{2}$. Cells were infected with $100 \mu \mathrm{l}$ of the virus-antibody mixtures for $1 \mathrm{~h}$, then $100 \mu \mathrm{l}$ of a $1 \times$ RPMI 1640 (Sigma-Aldrich, R6504), 1.5\% carboxymethylcellulose (Sigma-Aldrich, C4888) overlay was added without removing the inoculum. Cells were 
fixed 18 h after infection using 4\% PFA (Sigma-Aldrich) for 20 min. Foci were stained with a rabbit anti-spike monoclonal antibody (BS-R2B12, GenScript A02058) at $0.5 \mu \mathrm{g} \mathrm{ml}^{-1}$ in a permeabilization buffer containing $0.1 \%$ saponin (Sigma-Aldrich), 0.1\% BSA (Sigma-Aldrich) and 0.05\% Tween-20 (Sigma-Aldrich) in PBS. Plates were incubated with primary antibody overnight at $4{ }^{\circ} \mathrm{C}$, then washed with wash buffer containing $0.05 \%$ Tween-20 in PBS. Secondary goat anti-rabbit HRP conjugated antibody (Abcam ab205718) was added at $1 \mu \mathrm{g} \mathrm{ml}^{-1}$ and incubated for $2 \mathrm{~h}$ at room temperature with shaking. TrueBlue peroxidase substrate (SeraCare 5510-0030) was then added at $50 \mu \mathrm{l}$ per well and incubated for $20 \mathrm{~min}$ at room temperature. Plates were imaged in an ImmunoSpot Ultra-V S6-02-6140 Analyzer ELISPOT instrument with BioSpot Professional built-in image analysis (C.T.L).

\section{Statistics and fitting}

All statistics and fitting were performed in MATLAB v.2019b. Neutralization data were fit to:

$$
\mathrm{Tx}=\frac{1}{1+\left(D / \mathrm{ID}_{50}\right)}
$$

Here Tx is the number of foci normalized to the number of foci in the absence of plasma on the same plate at dilution $D$ and $\mathrm{ID}_{50}$ is the plasma dilution giving $50 \%$ neutralization. $\mathrm{FRNT}_{50}=1 / \mathrm{ID}_{50}$. Values of $\mathrm{FRNT}_{50}<1$ are set to 1 (undiluted), the lowest measurable value. Themostconcentrated plasmadilutionwas1:25and thereforeFRNT $\mathrm{N}_{50}<25$ were extrapolated. We have marked these values in Fig. 1c and calculate the fold-change $\mathrm{FRNT}_{50}$ either for the raw values or for values where FRNT $_{50}>25$ in Fig. $1 d$.

\section{Estimating vaccine efficacy from neutralization titres}

Previously, the fold reduction in neutralization was shown to correlate and predict vaccine efficacy against symptomatic infection with ancestral SARS-CoV- $2^{18}$, and more recently with variants of concern ${ }^{17}$ in data from randomized controlled trials. The model was used here to estimate the vaccine efficacy against Omicron based on the fold drop observed in this study applied to the randomized controlled trial data. In brief, vaccine efficacy (VE) was estimated based on the $\left(\log _{10}\right)$ fold drop in neutralization titre to Omicron $(f)$, and the $\left(\log _{10}\right)$ mean neutralization titre as a fold of the mean convalescent titre reported for BNT162b2 in phase I/II trials $(\mu)$ using the equation:

$$
\operatorname{VE}(\mu, f)=\int_{-\infty}^{\infty} N(x, \mu-f, \sigma) \frac{1}{1+\mathrm{e}^{-k\left(x-x_{50}\right)}} d x .
$$

Here, $N$ is the probability density function of a normal distribution with mean $\mu$ - $f$ and standard deviation $\sigma$, and $k$ and $x_{50}$ are the parameters of the logistic function relating neutralization to protection for the Pfizer BNT162b2 vaccine which were fitted from randomized controlled trial data: $\sigma=0.46, k=3$ and $x_{50}=\log _{10} 0.2$ for symptomatic infection $^{18}$. Importantly, $\mu=\log _{10} 2.4$ for trial participants vaccinated with two doses of BNT162b2, and $\mu=\log _{10} 12$ for vaccinated and boosted trial participants ${ }^{17,18}$.

\section{Informed consent and ethical statement}

Blood samples were obtained after written informed consent from hospitalized adults with PCR-confirmed SARS-CoV-2 infection and/ or vaccinated individuals who were enrolled in a prospective cohort study approved by the Biomedical Research Ethics Committee at the University of KwaZulu-Natal (reference BREC/00001275/2020). Use of residual swab sample was approved by the University of the Witwatersrand Human Research Ethics Committee (HREC) (ref. M210752).

\section{Reporting summary}

Further information on research design is available in the Nature Research Reporting Summary linked to this paper.

\section{Data availability}

Sequence of outgrown virus has been deposited in GISAID with accession EPI_ISL_7358094. Raw images of the data are available upon reasonable request.

\section{Code availability}

The sequence analysis and visualization pipeline are available on GitHub (https://github.com/nextstrain/ncov). Image analysis and curve fitting scripts in MATLAB v.2019b are available on GitHub (https:// github.com/sigallab/NatureMarch2021).

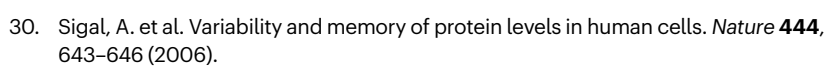

Acknowledgements This study was supported by the Bill and Melinda Gates award INV018944 (A.S.), National Institutes of Health award R01 Al138546 (A.S.), and South African Medical Research Council awards (A.S., T.d.O. and P.L.M.) and the UK Foreign, Commonwealth and Development Office and Wellcome Trust (grant no. $221003 /$ Z/2O/Z to P.L.M.). P.L.M. is also supported by the South African Research Chairs Initiative of the Department of Science and Innovation and the NRF (grant no. 98341). D.S.K., D.C. and M.P.D. are supported by NHMRC (Australia) Fellowship/Investigator grants. D.A. was supported by the European and Developing Countries Clinical Trials Partnership (EDCTP) Senior Fellowship (grant no. TMA2017SF-1960). The funders had no role in study design, data collection and analysis, decision to publish, or preparation of the manuscript.

Author contributions A.S., P.L.M. and T.d.O. and R.J.L. conceived the study. A.S., S.C., K.K., T.M.-G. and L.J. designed the study and experiments. A.v.G., P.L.M. and J.N.B. identified and provided the virus sample. S.-H.H. generated and provided plaque purified Beta variant virus. M.-Y.S.M., F.K., B.I.G., M.B., K.K. and Y.G. set up and managed the cohort and cohort data. S.C., L.J., K.K., T.M.-G., H.T., J.E.S., C.S., D.G.A., G.L., D.A., M.S., Y.G., Z.J. and K.R. performed experiments and sequence analysis with input from A.S., T.d.O., R.J.L. and J.M.B. D.S.K., D.C. and M.P.D. performed predictions of vaccine efficacy based on the data. A.S., S.C., P.L.M., T.d.O., L.J., K.K., W.H., S.S.A.K., D.S.K., M.P.D., J.N.B., R.J.L. and M.-Y.S.M. interpreted data. A.S., L.J., D.S.K., S.C., G.L., P.L.M. and M.P.D. prepared the manuscript with input from all authors.

Competing interests Salim S. Abdool Karim is a member of the COVID advisory panel for emerging markets at Pfizer. The authors declare no other competing interests.

Additional information

Supplementary information The online version contains supplementary material available at https://doi.org/10.1038/s41586-021-04387-1.

Correspondence and requests for materials should be addressed to Alex Sigal.

Peer review information Nature thanks the anonymous reviewer(s) for their contribution to the peer review of this work.

Reprints and permissions information is available at http://www.nature.com/reprints. 
A

Lentivirus

Serial dilution to $0.5 \mathrm{cell} /$ well

384 well plate

w/ ACE2

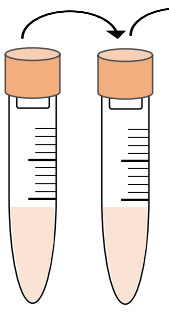

(3 weeks incubation)
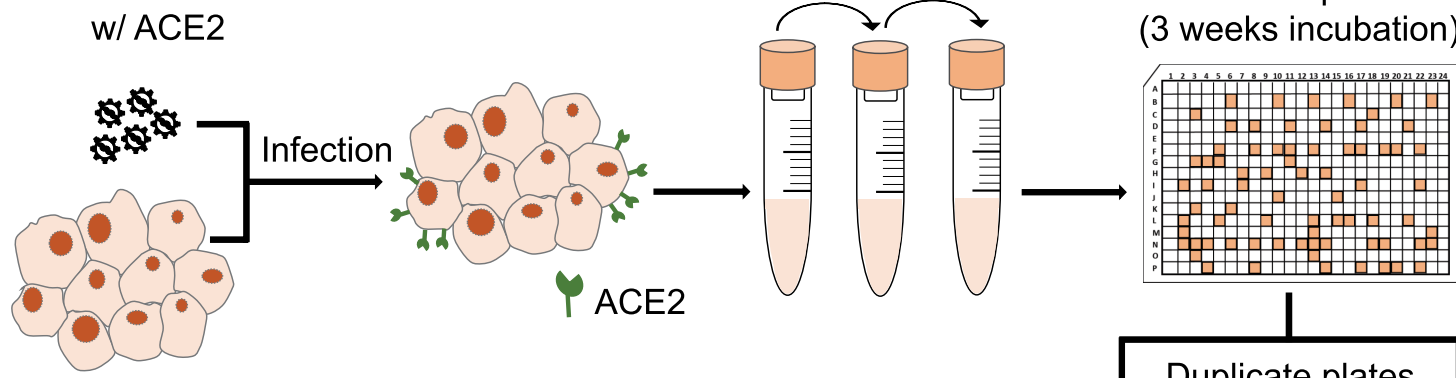

H1299

Confirm w/ spike pseudotyped lentivirus

B
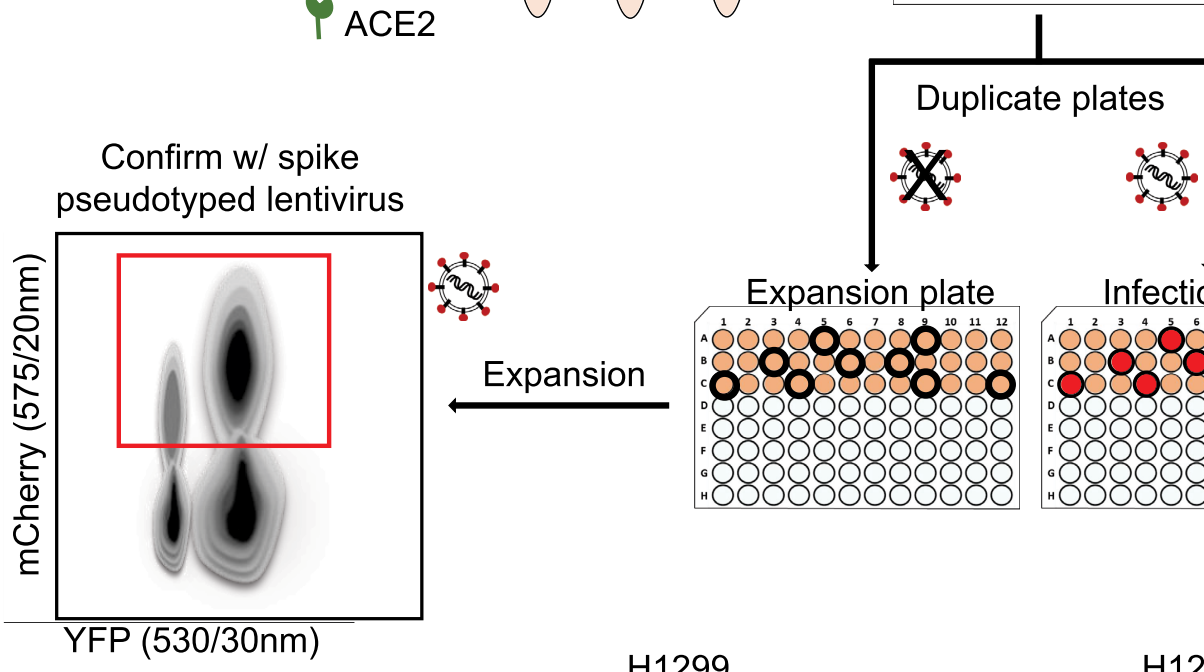

cells

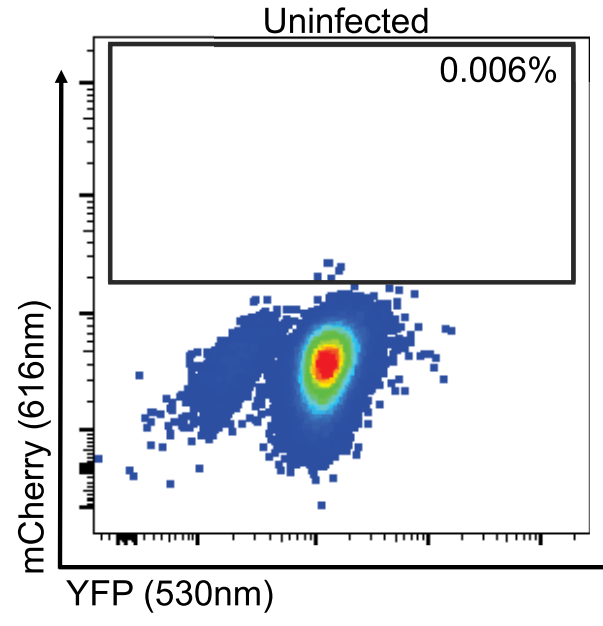

H1299

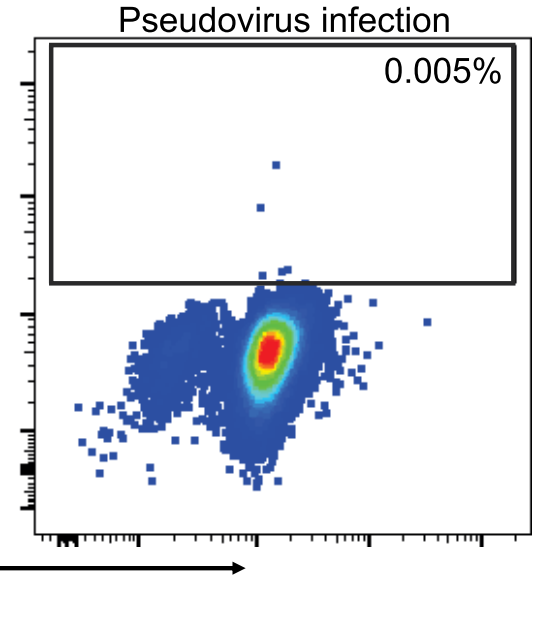

H1299-ACE2

Pseudovirus infection

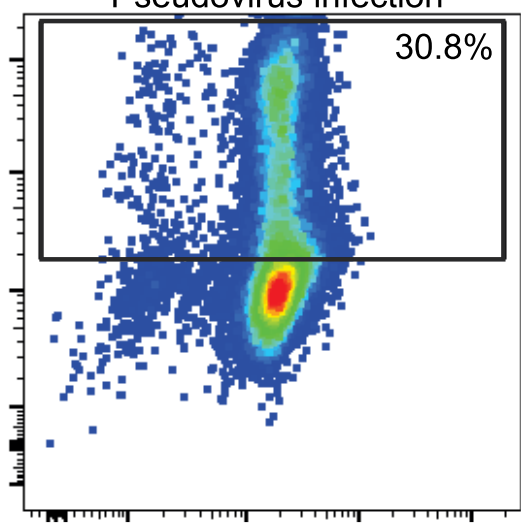

Extended Data Fig. 1 | Generation of H1299-ACE2 clonal cell line. (A) The H1299 human non-small cell lung carcinoma cell line with YFP labelled histone $\mathrm{H} 2 \mathrm{AZ}$ was spinfected with the pHAGE2-EF1a-Int-ACE2 lentivector. Cells were single cell cloned by limiting dilution in a 384-well plate. Clones were expanded into duplicate 96-well plates, where one plate was used to select infectable clones based on mCherry signal from infection with SARS-CoV-2 mCherry expressing spike pseudotyped lentivirus. Clones were chosen based on infectability and expanded from the non-infected replicate 96-well plate. (B) Flow cytometry of SARS-CoV-2 mCherry expressing spike pseudotyped lentivirus infection in H1299-ACE2 cells versus H1299 parental cells. 


\section{Article}

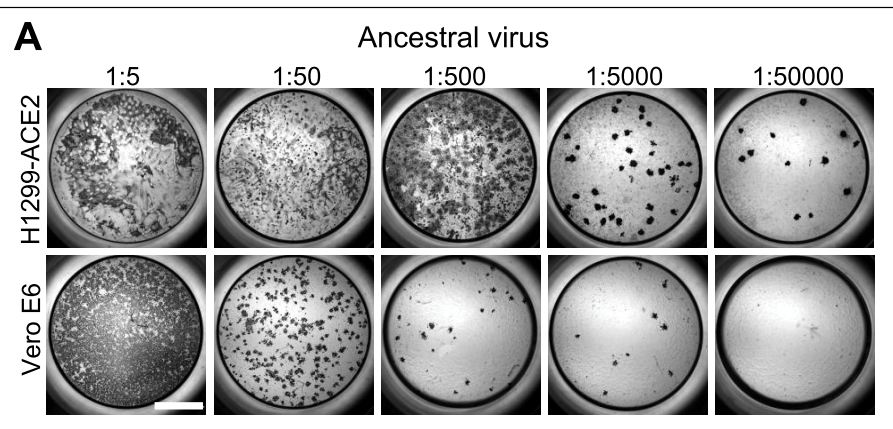

\section{B}

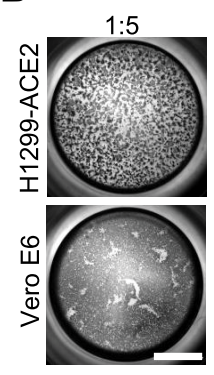

Beta virus
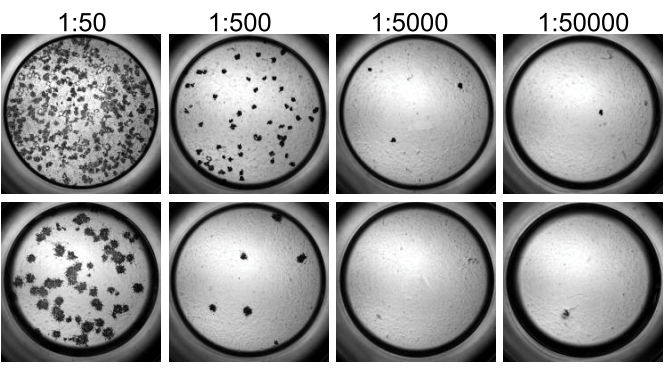

D Omicron virus (1:25)

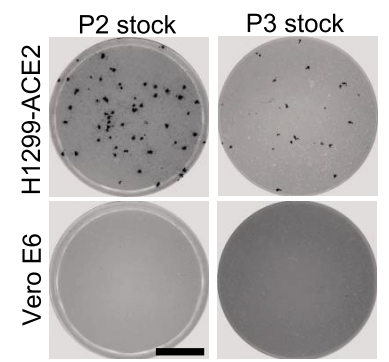

passage $2(\mathrm{P} 2)$ and passage $3(\mathrm{P} 3)$ stock, where $\mathrm{P} 3$ stock was generated by infection of $1 \mathrm{~mL}$ of cell-free $\mathrm{P} 2$ stock in $20 \mathrm{~mL}$ of Vero E6 cells seeded at $2 \times 10^{5}$ cells per $\mathrm{mL}$ and incubated over 4 days. Numbers above well images denote viral stock dilution. Scale bar is $2 \mathrm{~mm}$. and a focus forming assay was performed. (C) Focus forming assay with stock of Omicron virus isolate on H1299-ACE2 and Vero E6 cells. (D) Comparison of

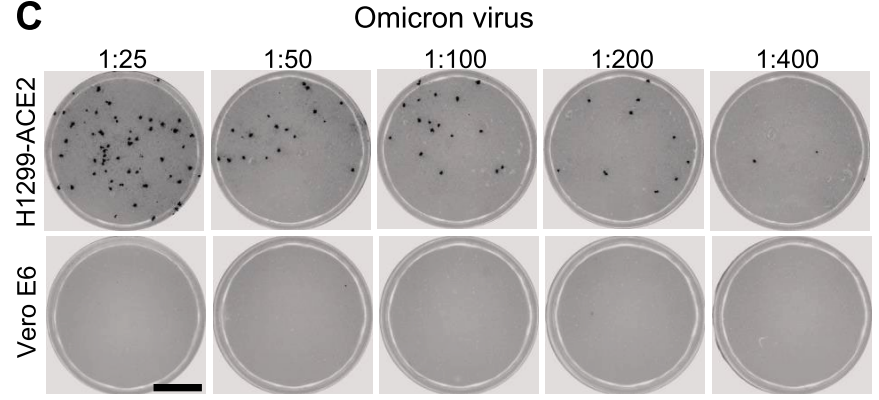

Extended Data Fig. 2 | Comparison of SARS-CoV-2 infection in H1299-ACE2 and Vero E6 cells. Both H1299-ACE2 and Vero E6 cells were infected with the 
A

H1299-ACE2

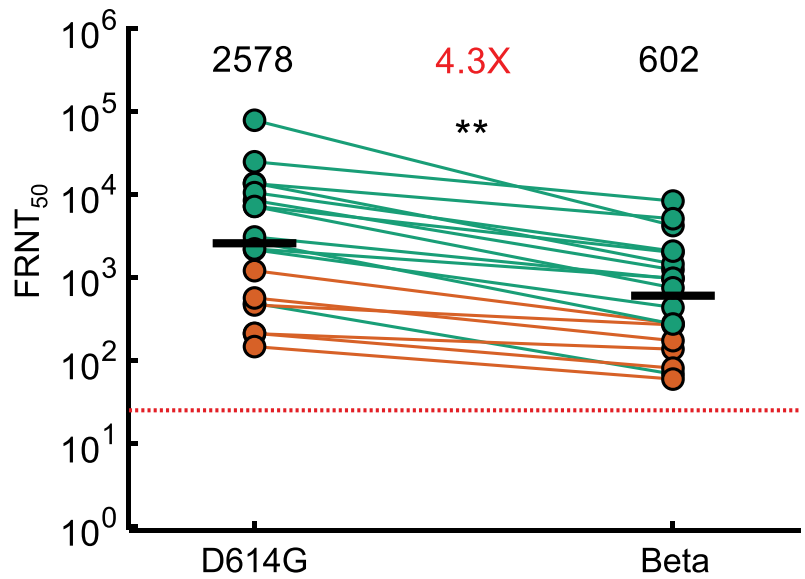

Extended Data Fig. 3 | Neutralization of the Beta variant by Pfizer BNT162b2 elicited immunity. Neutralization of the Beta variant virus compared to D614G ancestral virus in H1299-ACE2 (A) or Vero E6 cells (B) in participants vaccinated with BNT162b2 and infected by SARS-CoV-2 (green) or vaccinated only (orange). Numbers in black above each virus strain are geometric mean titers (GMT) of the reciprocal plasma dilution $\left(\mathrm{FRNT}_{50}\right.$ ) resulting in $50 \%$ reduction in the number of infection foci. Numbers in red
B

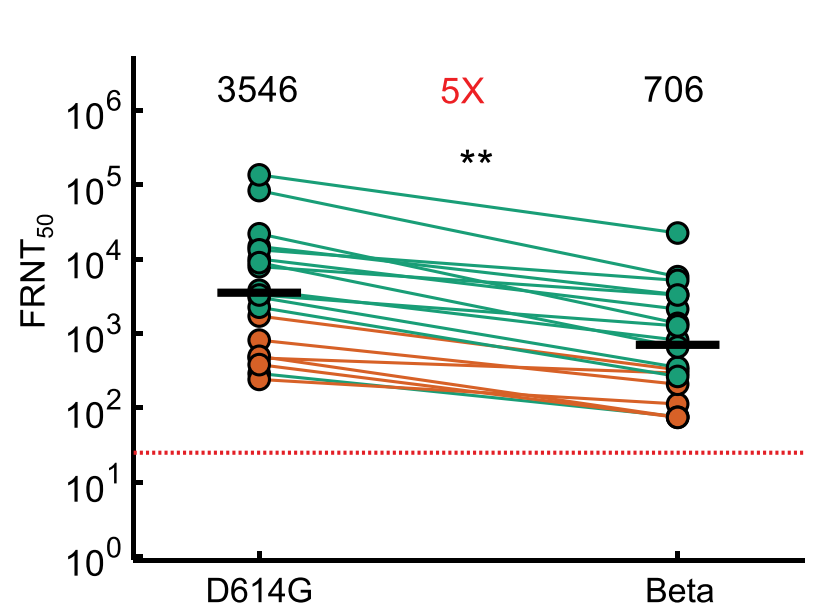

denote fold-change in GMT between virus strain on the left and the virus strain on the right of each panel. Red horizontal line denotes most concentrated plasma used. Samples were tested from the $n=19$ participants described in Table S2 and S3, where $n=6$ were vaccinated only and $n=13$ were vaccinated and previously infected. $\mathrm{p}=0.006$ for both $(\mathrm{A})$ and $(\mathrm{B})$ as determined by the Wilcoxon rank sum test. 


\section{Article}

Extended Data Table 1 | Codon frequency table

\begin{tabular}{|c|c|c|c|}
\hline $\begin{array}{l}\text { Amino Acid } \\
\text { Change }\end{array}$ & Nucleotide Change & Codon(s) Change & K032623_N67 \\
\hline \multirow[t]{2}{*}{ A67V } & $21762 C>T$ & 21761 & GCT - 0 \\
\hline & & $\mathrm{GCT}>\mathrm{GTT}$ & GTT - 133 \\
\hline \multirow[t]{2}{*}{ *H69_V70del } & 21766_21771delACATGT & 21766_21771ACATGT & ACATGT - 0 \\
\hline & & $>$ del & del -123 \\
\hline \multirow[t]{2}{*}{ T95I } & $21846 C>T$ & 21845 & ACT - 0 \\
\hline & & ACT $>$ ATT & ATT - 164 \\
\hline \multirow[t]{2}{*}{${ }^{*} \mathrm{G} 142 D$} & 21987_21989delGTG & 21987_21989GTG & GTG - 0 \\
\hline & & $>$ del & del -432 \\
\hline \multirow[t]{2}{*}{ "V143_Y145del } & 21990_21995delTTTATT & 21990_21995TTTATT & TTTATT - 0 \\
\hline & & $>$ del & del -432 \\
\hline \multirow[t]{2}{*}{ "L212I } & 22194_22196delATT & 22194_22196ATT & ATT - 0 \\
\hline & & $>$ del & del - 146 \\
\hline \multirow[t]{2}{*}{ “R214_D215 } & 22204_22205insGAGCCAGAA & 22204_22205GAGCCAGAA & WT - 37 \\
\hline & & $>$ ins & insGAGCCAGAA - 74 \\
\hline \multirow[t]{2}{*}{ G339D } & $22578 G>A$ & 22577 & GGT - 0 \\
\hline & & GGT>GAT & GAT - 255 \\
\hline \multirow[t]{2}{*}{ R346K } & $22599 G>A$ & 22598 & AGA - 1 \\
\hline & & $A G A>A A A$ & AAA - 250 \\
\hline \multirow[t]{2}{*}{ S371L } & $22674 \mathrm{C}>\mathrm{T}$ & 22674 & TCC - 0 \\
\hline & & $\mathrm{TCC}>\mathrm{CTC}$ & CTC - 152 \\
\hline \multirow[t]{2}{*}{ S373P } & $22679 \mathrm{~T}>\mathrm{C}$ & 22679 & TCA - 3 \\
\hline & & $\mathrm{TCA}>\mathrm{CCA}$ & CCA - 166 \\
\hline \multirow[t]{2}{*}{$S 375 F$} & $22686 \mathrm{C}>\mathrm{T}$ & 22685 & TCC - 0 \\
\hline & & $\mathrm{TCC}>\mathrm{TTC}$ & TTC - 160 \\
\hline \multirow[t]{2}{*}{ K417T } & $22813 G>T$ & 22811 & $A A G-3$ \\
\hline & & $A A G>A A T$ & AAT -934 \\
\hline \multirow[t]{2}{*}{ N440K } & $22882 T>G$ & 22880 & AAT -3 \\
\hline & & $A A T>A A G$ & AAG - 791 \\
\hline \multirow[t]{2}{*}{ G446S } & $22898 G>A$ & 22898 & GGT - 30 \\
\hline & & GGT>AGT & AGT -870 \\
\hline \multirow[t]{2}{*}{ T478K } & $22995 C>A$ & 22994 & ACA - 0 \\
\hline & & $A C A>A A A$ & AAA - 59 \\
\hline E484A & $23013 A>C$ & 23012 & GAA - 0 \\
\hline & & $G A A>G C A$ & GCA - 110 \\
\hline Q493R & $23040 A>G$ & 23039 & CAA - 0 \\
\hline & & $\mathrm{CAA}>\mathrm{CGA}$ & CGA - 128 \\
\hline G496S & $23048 G>A$ & 23048 & GGT - 0 \\
\hline & & GGT $>A G T$ & AGT - 150 \\
\hline Q498R & $23055 A>G$ & 23054 & CAA - 1 \\
\hline & & $\mathrm{CAA}>\mathrm{CGA}$ & CGA - 144 \\
\hline N501Y & $23063 A>T$ & 23063 & AAT - 0 \\
\hline & & AAT $>$ TAT & TAT - 209 \\
\hline Y505H & $23075 \mathrm{~T}>\mathrm{C}$ & 23075 & TAC - 1 \\
\hline & & $\mathrm{TAC}>\mathrm{CAC}$ & CAC - 261 \\
\hline T547K & $23202 C>A$ & 23201 & ACA - 0 \\
\hline & & $A C A>A A A$ & AAA - 777 \\
\hline D614G & $23403 A>G$ & 23402 & GAT - 1 \\
\hline & & GAT>GGT & GGT - 1803 \\
\hline H655Y & $23525 \mathrm{C}>\mathrm{T}$ & 23525 & CAT - 3 \\
\hline & & CAT $>\mathrm{TAT}$ & TAT - 1639 \\
\hline $\mathrm{N} 679 \mathrm{~K}$ & $23599 \mathrm{~T}>\mathrm{G}$ & 23597 & AAT - 1 \\
\hline & & $A A T>A A G$ & AAG - 682 \\
\hline P681H & $23604 C>A$ & 23603 & СCT - 0 \\
\hline & & $\mathrm{CCT}>\mathrm{CAT}$ & САТ - 535 \\
\hline Q954H & $24424 A>T$ & 24422 & CAA - 1 \\
\hline & & $\mathrm{CAA}>\mathrm{CAT}$ & CAT - 753 \\
\hline N969K & $24469 \mathrm{~T}>\mathrm{A}$ & 24467 & AAT - 0 \\
\hline & & AAT $>$ AAA & AAA - 1692 \\
\hline L981F & $24503 C>T$ & 24503 & CTT - 0 \\
\hline & & $\mathrm{CTT}>\mathrm{TTT}$ & TTT - 1797 \\
\hline
\end{tabular}

This table shows the amino acid change, the nucleotide position of the genome, codon change and the frequency of the codon on the assembled genome. ${ }^{*}$ Only deletions or insertion where the adjacent codon was preserved were counted; WT - Wild Type, i.e reads without the insertion. 
Extended Data Table 2 | Summary table of participants

\begin{tabular}{lccc}
\hline & All & Vaccinated only & Infected and vaccinated \\
\hline Number of Participants & 19 & 6 & 13 \\
Age (years) & $52(39-67)$ & $54(36-71)$ & $51(45-63)$ \\
$\begin{array}{l}\text { Days post-vaccination } \\
\text { Days post-infection }\end{array}$ & $26(14-33)$ & $14.5(8.5-37.5)$ & $28(18-32)$ \\
$\begin{array}{l}\text { Days post-infection to vaccination } \\
\text { Date range of symptom onset }\end{array}$ & & & $379(127-468)$ \\
Male sex & 7 & & $353(114-444)$ \\
\hline
\end{tabular}

All values are median (IQR) and inclusive of all samples used (early and late timepoints for 2 participants). 


\section{Article}

Extended Data Table 3 | Participant information per sample

\begin{tabular}{|c|c|c|c|c|c|c|c|c|c|}
\hline Sample & Participant & Age & Sex & $\begin{array}{c}\text { Days post } 2^{\text {nd }} \\
\text { vaccination dose }\end{array}$ & $\begin{array}{l}\text { Days diagnostic } \\
\text { swab to sample }\end{array}$ & $\begin{array}{c}\text { Date symptom onset or } \\
\text { diagnostic test }\end{array}$ & Infecting virus* & $\begin{array}{l}\text { FRNT }_{50} \\
\text { D614G }\end{array}$ & $\begin{array}{l}\text { FRNT }_{50} \\
\text { Omicron }\end{array}$ \\
\hline 1 & 1 & $60-69$ & $\mathrm{~F}$ & 10 & - & - & - & 196 & 10.8 \\
\hline 2 & 2 & $70-79$ & M & 10 & - & - & - & 463 & 26.1 \\
\hline 3 & 2 & $70-79$ & M & 45 & - & - & - & 205 & 14.6 \\
\hline 4 & 3 & $30-39$ & M & 14 & - & - & - & 485 & 31.1 \\
\hline 5 & 4 & $70-79$ & $\mathrm{~F}$ & 10 & - & - & - & 199 & 15.4 \\
\hline 6 & 4 & $70-79$ & $\mathrm{~F}$ & 48 & - & - & - & 76.8 & 1.0 \\
\hline 7 & 5 & $30-39$ & $\mathrm{~F}$ & 10 & - & - & - & 1102 & 51.9 \\
\hline 8 & 6 & $30-39$ & $\mathrm{~F}$ & 33 & - & - & - & 151 & 4.6 \\
\hline 9 & 7 & $40-49$ & $\mathrm{~F}$ & 14 & 458 & Jul-2020 & Ancestral & 10447 & 681 \\
\hline 10 & 8 & $60-69$ & $\mathrm{~F}$ & 63 & 468 & Jul-2020 & Ancestral & 7468 & 414 \\
\hline 11 & 9 & $20-29$ & $\mathrm{~F}$ & 31 & 487 & Aug-2020 & Ancestral & 2153 & 190 \\
\hline 12 & 10 & $20-29$ & M & 37 & 493 & Jul-2020 & Ancestral & 2697 & 121 \\
\hline 13 & 11 & $60-69$ & $\mathrm{~F}$ & 28 & 378 & Jul-2020 & Ancestral & 54823 & 892 \\
\hline 14 & 12 & $60-69$ & M & 26 & 379 & Jul-2020 & Ancestral & 47023 & 1550 \\
\hline 15 & 13 & $40-49$ & $\mathrm{~F}$ & 32 & 479 & Aug-2020 & Ancestral & 13517 & 955 \\
\hline 16 & 14 & $50-59$ & $M$ & 30 & 370 & Sep-2020 & Ancestral & 11590 & 681 \\
\hline 17 & 15 & $40-49$ & $\mathrm{~F}$ & 22 & $456^{* *}$ & Jun-2020** & Ancestral/Delta & 664 & 5.0 \\
\hline 18 & 16 & $40-49$ & M & 18 & 83 & Jul-2021*** & Delta & 10511 & 749 \\
\hline 19 & 17 & $70-79$ & M & 37 & 8 & Jul-2021 & Delta & 3074 & 138 \\
\hline 20 & 18 & $50-59$ & $\mathrm{~F}$ & 13 & 127 & Jul-2021*** & Delta & 2205 & 385 \\
\hline 21 & 19 & $60-69$ & $\mathrm{~F}$ & 14 & 103 & Jul-2021 & Delta & 7160 & 174 \\
\hline
\end{tabular}

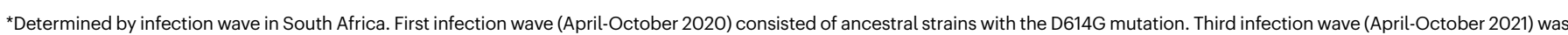

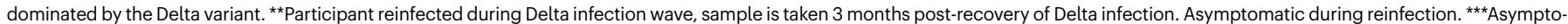
matic. 


\section{Reporting Summary}

Nature Portfolio wishes to improve the reproducibility of the work that we publish. This form provides structure for consistency and transparency in reporting. For further information on Nature Portfolio policies, see our Editorial Policies and the Editorial Policy Checklist.

\section{Statistics}

For all statistical analyses, confirm that the following items are present in the figure legend, table legend, main text, or Methods section.

$\mathrm{n} / \mathrm{a} \mid$ Confirmed

$\bigotimes$ The exact sample size $(n)$ for each experimental group/condition, given as a discrete number and unit of measurement

$\square$ A statement on whether measurements were taken from distinct samples or whether the same sample was measured repeatedly

The statistical test(s) used AND whether they are one- or two-sided

Only common tests should be described solely by name; describe more complex techniques in the Methods section.

Х $\square$ A description of all covariates tested

Х $\square$ A description of any assumptions or corrections, such as tests of normality and adjustment for multiple comparisons

$\triangle$ A full description of the statistical parameters including central tendency (e.g. means) or other basic estimates (e.g. regression coefficient)

AND variation (e.g. standard deviation) or associated estimates of uncertainty (e.g. confidence intervals)

For null hypothesis testing, the test statistic (e.g. $F, t, r$ ) with confidence intervals, effect sizes, degrees of freedom and $P$ value noted

Give $P$ values as exact values whenever suitable.

Х $\square$ For Bayesian analysis, information on the choice of priors and Markov chain Monte Carlo settings

$\triangle \square$ For hierarchical and complex designs, identification of the appropriate level for tests and full reporting of outcomes

$\triangle(\square$ Estimates of effect sizes (e.g. Cohen's $d$, Pearson's $r$ ), indicating how they were calculated

Our web collection on statistics for biologists contains articles on many of the points above.

\section{Software and code}

Policy information about availability of computer code

Data collection No code used

Data analysis All statistics and fitting were performed using MATLAB v.2019b.

For manuscripts utilizing custom algorithms or software that are central to the research but not yet described in published literature, software must be made available to editors and

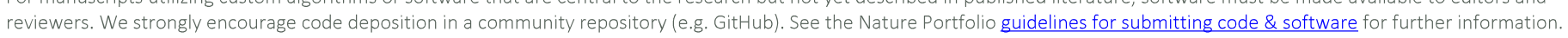

\section{Data}

Policy information about availability of data

All manuscripts must include a data availability statement. This statement should provide the following information, where applicable:

- Accession codes, unique identifiers, or web links for publicly available datasets

- A description of any restrictions on data availability

- For clinical datasets or third party data, please ensure that the statement adheres to our policy 
Please select the one below that is the best fit for your research. If you are not sure, read the appropriate sections before making your selection.

$\bigotimes$ Life sciences $\quad \square$ Behavioural \& social sciences $\quad \square$ Ecological, evolutionary \& environmental sciences

For a reference copy of the document with all sections, see nature.com/documents/nr-reporting-summary-flat.pdf

\section{Life sciences study design}

All studies must disclose on these points even when the disclosure is negative.

Sample size Sample size was not pre-determined. We used all the samples we had available which met the inclusion/exclusion criteria.

Data exclusions We excluded samples from PfizerBNT162b2 vaccinated participants who were previously infected with the Beta variant since we wanted to compare to the Omicron to Beta virus neutralization. We excluded samples positive for SARS-CoV-2 nucleocapsid (ie previously infected) where we could not determine the infecting variant/strain by a time of infection.

Replication Repeated in an independent experiment on a different day. Geometric mean of replicate samples was used.

Randomization Groups were determined based on whether

Blinding No blinding.

\section{Reporting for specific materials, systems and methods}

We require information from authors about some types of materials, experimental systems and methods used in many studies. Here, indicate whether each material, system or method listed is relevant to your study. If you are not sure if a list item applies to your research, read the appropriate section before selecting a response.

Materials \& experimental systems

n/a Involved in the study

$\square$ Antibodies

Х $\square$ Palaeontology and archaeology

\ $\square$ Animals and other organisms

$\square$ \uman research participants

\ $\square$ Clinical data

$\bigotimes \square$ Dual use research of concern $\square \bigotimes$ Eukaryotic cell lines

\begin{tabular}{l|l}
\multicolumn{2}{l}{ Methods } \\
\hline n/a & Involved in the study \\
$\square$ & $\square$ ChIP-seq \\
$\square$ & $\bigotimes$ Flow cytometry \\
$\square$ & $\square$ MRI-based neuroimaging
\end{tabular}

\section{Antibodies}

Antibodies used

Foci were stained with a rabbit anti-spike monoclonal antibody (BS-R2B12, GenScript A02058) at $0.5 \mu \mathrm{g} / \mathrm{mL}$. Secondary goat antirabbit horseradish peroxidase (Abcam ab205718) antibody was added at $1 \mu \mathrm{g} / \mathrm{mL}$

Validation

Information sheet for A02058 at https://www.genscript.com/antibody/A02058-

MonoRab_SARS_CoV_2_Spike_S1_Antibody_BS_R2B12_mAb_Rabbit.html. Information sheet for ab205718: https://

www.abcam.com/goat-rabbit-igg-hl-hrp-ab205718.html

\section{Eukaryotic cell lines}

Policy information about cell lines

Cell line source(s)

Authentication

Mycoplasma contamination

Commonly misidentified lines (See ICLAC register)
Vero E6 cells (ATCC CRL-1586) obtained from Cellonex in South Africa. The H1299-E3 cell line was derived from H1299 (CRL-5803) as described in (2) and Figure S1. H1299 cells were a gift from M. Oren, Weizmann Institute of Science.

\section{Cell lines have not been authenticated.}

The cell lines have been tested for mycoplasma contamination and are mycoplasma negative.

None. 
Policy information about studies involving human research participants

Population characteristics

Participant characteristics are summarized in Table S1 and listed per participant in Table S2.

Recruitment

Blood samples were obtained from hospitalized adults with PCR-confirmed SARS-CoV- 2 infection and/or vaccinated individuals who were enrolled in a prospective cohort study approved by the Biomedical Research Ethics Committee at the University of KwaZulu-Natal.

Ethics oversight

Study approved by the Biomedical Research Ethics Committee at the University of KwaZulu-Natal (reference BREC/00001275/2020). Use of residual swab sample was approved by the University of the Witwatersrand Human Research Ethics Committee (HREC) (ref. M210752).

Note that full information on the approval of the study protocol must also be provided in the manuscript.

\section{Flow Cytometry}

Plots

Confirm that:

W The axis labels state the marker and fluorochrome used (e.g. CD4-FITC).

Х The axis scales are clearly visible. Include numbers along axes only for bottom left plot of group (a 'group' is an analysis of identical markers).

\All plots are contour plots with outliers or pseudocolor plots.

\A numerical value for number of cells or percentage (with statistics) is provided.

\section{Methodology}

Sample preparation

Instrument

Software

Cell population abundance

Gating strategy

$\bigotimes$ Tick this box to confirm that a figure exemplifying the gating strategy is provided in the Supplementary Information.
Plasma was separated from EDTA-anticoagulated blood by centrifugation at $500 \mathrm{rcf}$ for $10 \mathrm{~min}$ and stored at $-80^{\circ} \mathrm{C}$. Aliquots of plasma samples were heat-inactivated at $56^{\circ} \mathrm{C}$ for $30 \mathrm{~min}$ and clarified by centrifugation at $10,000 \mathrm{rcf}$ for $5 \mathrm{~min}$.

Plates were imaged in an ImmunoSpot Ultra-V S6-02-6140 Analyzer ELISPOT instrument with BioSpot Professional built-in image analysis (C.T.L).

BioSpot Professional built-in image analysis (C.T.L).

H1299-E3 clone was previously generated and described. Abundance of infected cells with lentiviral infection was 30\%/

H1299-E3 clone was previously generated and described. Gating was based on FSC/SSC for live cells, then uninfected cells were used to determine mCherry positive gating. 